\title{
SANO97-0147C \\ CONF- $9705139--1$ \\ COMPARISON OF LUMPED-ELEMENT AND TRANSMISSION-LINE MODELS FOR THICKNESS-SHEAR-MODE QUARTZ RESONATOR SENSORS
}

\author{
R. W. Cernosek ${ }^{\dagger}$, S. J. Martin ${ }^{\dagger}$, A. R. Hillman ${ }^{\ddagger}$, and H. L. Bandey ${ }^{\dagger}$ \\ ${ }^{\dagger}$ Sandia National Laboratories, Microsensor Research and Development Dept., \\ P.O. Box 5800, MS 1425, Albuquerque, NM 87185-1425, USA RECEIVED \\ ${ }^{\ddagger}$ University of Leicester, Department of Chemistry, \\ University Road, Leicester, LE1 7RH, United Kingdom

\begin{abstract}
Both a transmission-line model and its simpler variant, a lumped-element model, can be used to predict the responses of a thickness-shear-mode quartz resonator sensor. Relative deviations in the parameters computed by the two models (shifts in resonant frequency and motional resistance) do not exceed $3 \%$ for most practical sensor configurations operating at the fundamental resonance. If the ratio of the load surface mechanical impedance to the quartz shear characteristic impedance does not exceed 0.1 , the lumped-element model always predicts responses within $1 \%$ of those for the transmission-line model.

\section{Introduction}

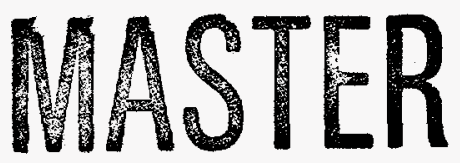

The thickness-shear-mode (TSM) quartz resonator has become an extremely useful tool for measuring material properties and monitoring chemical processes. Various media in contact with the vibrating quartz surface create mechanical perturbations that alter the system resonance characteristics; shifts occur in the resonant frequency and motional resistance from that of the bare crystal. A key component in developing sensors based on the TSM resonators is to identify the relationship between the measurable electrical responses and the surface loading. Then properties of interest, such as accumulated mass, liquid density-viscosity, or film elastic modulus, can be extracted and studied.
\end{abstract}

One methodology is to treat the sensor system as an acoustic transmission line having one driven piezoelectric layer (the quartz crystal) and one or more surface mechanical loads consisting of lumped impedance elements or non-piezoelectric distributed layers [1-3]. This treatment produces a generalized and elegant mathematical description of the surface-loaded resonator. In practice, it is common to investigate TSM resonator sensors using impedance or admittance analysis. The automatic network analyzer (ANA) acquires the appropriate spectrum and 


\section{DISCLAMEER}

Portions of this document may be illegible in electronic image products. Images are produced from the best available original document. 
the mathematical model is used to extract the sensed parameters. However, during data analysis, applying a full transmission-line model (TLM) to the resonator sensing system is often cumbersome and time consuming. A simpler approach is to use a lumped-element model (LEM) that represents mechanical interactions by their equivalent electrical circuit components $[4,5]$. The LEM is nothing more than a reduced version of the TLM that assumes surface load impedances are small compared to the shear mechanical impedance of the quartz and that sensor operating frequencies are always near mechanical resonance. The lumped-element representation not only simplifies mathematical analysis but also provides an intuitive means of understanding load interactions.

In this paper we briefly discuss the theoretical development of the transmission-line model for a TSM resonator sensor and the simplifying assumptions that lead to the lumped-element model. Each model is then used to compute the expected responses for typical sensing applications. We are interested in the shift in the series resonance frequency and the increase in resonance damping due to surface loading since these are "measurable" parameters in most sensor systems. Relative deviations in these two parameters establishes a quantitative means for determining the utility of the LEM.

\section{TSM Resonator Transmission-Line Theory}

A TSM resonator consists of a thin disk of AT-cut quartz with metal electrodes deposited on both. Due to the piezoelectric properties and crystal orientation of the quartz, application of a voltage between the electrodes results in shear deformation of the crystal. At the natural mechanical resonances, a standing wave pattern is generated across the crystal thickness with displacement maxima occurring at the faces. Electrical excitation can produce several resonant modes (harmonics) each with a standing wave displacement profile. (See Figs. 3 and 7 for crosssectional views of fundamental mode displacement in loaded crystal resonators.) Resonance characteristics of the crystal, primarily the resonant frequency (f) and quality factor $(Q)$, are perturbed by materials that mechanically load the quartz surface. Extracting the mechanical properties of the load from electrical measurements is the sensing goal.

The TSM resonator is a one-port electrical device whose piezoelectric excitation and acoustic transmission properties are best represented by the three-port Mason model (see Fig. 1) [1]. The electrical port in Fig. 1 is characterized by a $1: \mathrm{N}^{\prime}$ transformer that electromechanically couples the applied voltage (at A-B in Fig. 1 ) to the 
quartz shear displacement (at C-D). Shear acoustic waves propagate between the surfaces of the quartz crystal, the two acoustic ports in the three-port model. Impedance changes at the quartz surfaces will transmit and/or reflect the acoustic energy, depending on the impedance mismatch. In typical sensing applications, one surface of the crystal is tension-free and, thus, has a zero impedance as illustrated in Fig. 1. At the loaded sensor surface, a generic mechanical load impedance, $Z_{I}$, is encountered. This load can be a single impedance element, a transmission line element such as a non-piezoelectric layer, or combinations of the two.

Vibrational behavior of the quartz crystal and the surface load can be treated using a one-dimensional transmission-line model and solving for the wave equation in each medium. A detailed mathematical development of the transmission-line theory is given by several authors [1-3]. The complex electrical input impedance for the quartz resonator described by the model in Fig. 1 is

$$
\begin{aligned}
Z & =Z_{A B}=\frac{1}{j \omega C_{0}}+j X+\frac{1}{\left(N^{\prime}\right)^{2}} Z_{C D} \\
& =\frac{1}{j \omega C_{0}}\left[1-\frac{K^{2}}{\alpha} \frac{2 \tan \left(\frac{\alpha}{2}\right)-j \zeta}{1-j \zeta \cot (\alpha)}\right] .
\end{aligned}
$$

In Eq. (1), $Z_{C D}$ is the acoustic impedance at $C-D$ in Fig. 1, [the capacitance $C_{0}$, electrical reactance $X$, and transformer turns ratio $\mathrm{N}^{\prime}$ are as shown in Fig. $\left.1 ;\right] \omega=2 \pi f$ is the oscillation frequency, $\mathrm{K}^{2}$ is the complex electromechanical coupling factor for lossy quartz, $\alpha$ is the complex acoustic wave phase shift across the lossy quartz, and $\zeta=Z_{I} / Z_{q}$ where $Z_{L}$ is the surface load mechanical impedance and $Z_{q}=\left(\rho_{q} \mu_{q}\right)^{1 / 2}$ is the quartz characteristic impedance with $\rho_{q}$ and $\mu_{q}$ the quartz density and shear elastic constant. (See Table I for quartz parameters.) Since quartz is a low-loss material, the lossless parameters $K_{0}^{2}$ and $\alpha_{0}=\omega h_{q}\left(\rho_{q} / \mu_{q}\right)^{1 / 2}$, with $h_{q}$ the quartz thickness, often can be used in Eq. (1).

The electrical impedance in Eq. (1) can be represented as a static capacitance $C_{0}$ in parallel with a motional impedance, $Z_{\mathrm{m}}$, arising from mechanical resonance. Rearranging Eq. (1) gives the motional impedance term:

$$
Z_{m}=\frac{1}{j \omega C_{0}}\left[\frac{1-j \zeta \cot (\alpha)}{\frac{K^{2}}{\alpha}\left(2 \tan \left(\frac{\alpha}{2}\right)-j \zeta\right)}-1\right],
$$


which can be further reduced to

$$
\begin{aligned}
Z_{m} & =\frac{1}{j \omega C_{0}}\left[\frac{\frac{\alpha}{K^{2}}}{2 \tan \left(\frac{\alpha}{2}\right)}-1\right]+\frac{1}{\omega C_{0}} \frac{\alpha \zeta}{4 K^{2}}\left[1-\frac{j \zeta}{2 \tan (\alpha / 2)}\right]^{-1} \\
& =Z_{m}^{0}+Z_{m}^{L} .
\end{aligned}
$$

The two terms in Eq. (3) describe the motional impedance for the unperturbed quartz resonator, $\mathrm{Z}_{\mathrm{m}}{ }^{0}$, and the added motional impedance created by the surface load, $Z_{\mathrm{m}}^{\mathrm{I}}$.

It is more straightforward to compute and analyze the input admittance of a quartz resonator sensor instead of its impedance; from Eqs. (1) and (7) the admittance is given by

$$
Y(\omega)=\frac{1}{Z(\omega)}=j \omega C_{0}^{*}+\frac{1}{Z_{m}^{0}+Z_{m}^{L}}
$$

where $C_{0}{ }^{\circ}=C_{0}+C_{p}$, with $C_{p}$ an added parasitic capacitance accounting for packaging, connection, etc. At series resonance the motional elements, $Z_{m}{ }^{0}$ and $Z_{m}{ }^{L}$, control the oscillation while the static capacitance, $C_{0}^{*}$, is shunted. This occurs at a frequency where the reactive component of the motional impedance is zero, and thus $|\operatorname{Re}(\mathrm{Y})|$ is at maximum. For the purpose of comparing sensor application responses, we focus on the shift (or changes) in two measurable parameters: the series resonant frequency, $f_{s}=\omega_{3} / 2 \pi$, and the total motional resistance, $R$. The shift in these two quantities can be extracted directly from the admittance versus frequency analysis using

$$
\Delta f_{s}=\left(f_{s}\right)^{L}-\left(f_{s}\right)^{0}
$$

where $f_{s}$ occurs at the maximum value of $|\operatorname{Re}(Y)|$,

and

$$
\Delta \mathrm{R}=\frac{1}{\left(|\operatorname{Re}(\mathrm{Y})|_{\max }\right)^{\mathrm{I}}}-\frac{1}{\left(|\operatorname{Re}(\mathrm{Y})|_{\max }\right)^{0}} .
$$

The superscripts $L$ and 0 refer to the surface loaded and unperturbed resonator responses, respectively.

\section{The Unperturbed Resonator}

From Eq. (3), the motional impedance associated with an unperturbed resonator is given by 


$$
Z_{m}^{0}=\frac{1}{j \omega C_{0}}\left[\frac{\frac{\alpha}{K^{2}}}{2 \tan \left(\frac{\alpha}{2}\right)}-1\right]
$$

At series resonance, the imaginary part of $\mathrm{Eq}$. (7) is zero and the resonant frequency, $\omega_{s}$, satisfies the transcendental equation

$$
\omega_{\mathrm{s}}=\frac{2 \mathrm{~K}_{0}^{2}}{\mathrm{~h}_{\mathrm{q}}} \sqrt{\frac{\mu_{\mathrm{q}}}{\rho_{\mathrm{q}}}} \tan \left(\omega_{\mathrm{s}} \frac{\mathrm{h}_{\mathrm{q}}}{2} \sqrt{\frac{\rho_{\mathrm{q}}}{\mu_{\mathrm{q}}}}\right) .
$$

For resonator operation at frequencies near mechanical resonance, several approximations are made to simplify Eq. (7) into a series of lumped elements:

$$
Z_{m}^{0} \approx R_{1}+j \omega L_{1}+\frac{1}{j \omega C_{1}}
$$

This expression describes the motional impedance for the Butterworth-Van Dyke (BVD) equivalent circuit of an unperturbed resonator (see Fig. 2). The circuit elements for the BVD model are [4]

$$
\begin{gathered}
\mathrm{C}_{0}=\frac{\varepsilon_{\mathrm{q}} \mathrm{A}}{\mathrm{h}_{\mathrm{q}}}, \\
\mathrm{R}_{1}=\frac{(\mathrm{N} \pi)^{2}}{8 \mathrm{~K}_{0}^{2} \mathrm{C}_{0}} \frac{\eta_{\mathrm{q}}}{\mu_{\mathrm{q}}}, \\
\mathrm{C}_{1}=\frac{8 \mathrm{~K}_{0}^{2} \mathrm{C}_{0}}{(\mathrm{~N} \pi)^{2}}, \\
\mathrm{~L}_{1}=\frac{1}{\omega_{\mathrm{s}}^{2} \mathrm{C}_{1}},
\end{gathered}
$$

where $\varepsilon_{\mathrm{q}}$ is the quartz permittivity, $A$ is the effective area of the electrodes on the crystal, $\eta_{\mathrm{q}}$ is the quartz viscosity. and $N$ is the harmonic resonance of the quartz, $N=1,3,5, \ldots$. The series resonant frequency is given by [1]

$$
\omega_{\mathrm{s}} \approx \frac{\mathrm{N} \pi}{\mathrm{h}_{\mathrm{q}}} \sqrt{\frac{\mu_{\mathrm{q}}}{\rho_{\mathrm{q}}}} \sqrt{1-\frac{8 \mathrm{~K}_{0}^{2}}{(\mathrm{~N} \pi)^{2}}} .
$$

Eqs. (8) and (14) give the predicted series resonant frequencies for the full transmission-line characterization and the lumped-element approximation, respectively. The deviation between these frequencies (determined empirically) is approximately $5 \mathrm{ppm}$. Thus, the lumped-element BVD model can be used to represent the unperturbed resonator 
in all sensor calculations.

\section{The Surface Loaded Resonator}

Based on Eq. (3), the motional impedance of the quartz resonator with a surface mechanical load can be treated as the sum of two impedance elements: one describing the motion of the unperturbed quartz crystal and one describing the interaction with the load. In the previous section, we determined the unperturbed resonator can be represented by the simple BVD equivalent circuit with a high degree of computational precision. The loaded quartz resonator sensor can then be modeled using the modified BVD equivalent circuit shown in Fig. 2 [4,5]. The load motional impedance element in Fig. 2a is extracted from Eq. (3) and given by

$$
Z_{m}^{L}=\frac{1}{\omega C_{0}} \frac{\alpha \zeta}{4 K^{2}}\left[1-\frac{j \zeta}{2 \tan (\alpha / 2)}\right]^{-1}
$$

In general, the real and imaginary parts of $Z_{m}{ }^{I}$ can be determined and the motional resistance, $R_{2}$; and motional inductance, $\mathrm{L}_{2}$, of the load can be computed as illustrated in Fig. $2 b$. These two lumped components represent the power dissipation and the energy storage, respectively, in the load. For some surface mechanical loads, closed forms of $R_{2}$ and $L_{2}$ can be computed; for others, analytical extraction is not possible and they must be determined empirically.

Close inspection of Eq. (15) indicates that for small surface loading, the last term in brackets is much less than one so that

$$
\frac{\left|Z_{I}\right|}{Z_{q}} \ll 2 \tan \left(\frac{\alpha}{2}\right)
$$

Then for operation near the series resonance frequency, $\omega \approx \omega_{3}, \alpha \approx N \pi$, and Eq. (15) reduces to [3]

$$
Z_{m}^{L}=\frac{N \pi}{4 K_{0}^{2} \omega_{s} C_{0}}\left(\frac{Z_{L}}{Z_{q}}\right) \text {, }
$$

which is the load motional impedance for the lumped-element model. Under certain conditions, it is evident that Eq. (16) is valid and the TLM will reduce to the LEM. In many sensor applications, $Z_{\mathscr{L}}$ is often several orders of magnitude smaller than $Z_{q}\left(Z_{q}=\left[\rho_{q} \mu_{q}\right]^{1 / 2}=8.84 \times 10^{5} \mathrm{~g} \cdot \mathrm{cm}^{-2} \cdot \mathrm{s}^{-1}\right.$ using the values in Table $\left.\mathrm{I}\right)$. At the same time, when $\omega \approx \omega_{\mathrm{s}}\left(\Delta \omega_{\mathrm{s}}\right.$ small $)$ and $\alpha \approx \mathrm{N} \pi, \tan (\alpha / 2)$ becomes extremely large. However, as $Z_{\mathrm{L}}$ increases, $\left|Z_{\mathrm{L}}\right| / Z_{\mathrm{q}}$ also - 
increases, while $\Delta \omega_{s}$ becomes larger and $\tan (\alpha / 2)$ decreases.

Since the quantities on both sides of the inequality in Eq. (16) are coupled, it is difficult to determine analytically when the surface loading becomes too large to preclude use of the simpler lumped-element model. Therefore, the deviations between the TLM and the LEM were determined computationally for several types of surface loads. Analytical expressions for $Z_{L}$ were substituted into Eqs. (15) for the TLM and Eq. (17) for the LEM. In both cases, $Z_{m}{ }^{L}$ was then used in Eq. (4), with $Z_{m}{ }^{0}$ as described by Eq. (9). All values for the unperturbed resonator were kept constant as listed in Table II. The measurable parameters of interest, $\Delta f_{3}$ and $\Delta R$ (same as $R_{2}$ in Fig. 2b), were computed from Eqs. (5) and (6) as part of the admittance analysis. The final relative deviations between the model predictions were determined from

and

$$
\begin{aligned}
& \frac{\delta\left(\Delta f_{s}\right)}{\Delta f_{s}}=\frac{\Delta f_{s}(T L M)-\Delta f_{s}(L E M)}{\Delta f_{s}(T L M)}, \\
& \frac{\delta(\Delta R)}{\Delta R}=\frac{\Delta R(T L M)-\Delta R(L E M)}{\Delta R(T L M)}
\end{aligned}
$$

\section{Mass Loading}

An ideal mass layer, shown in Fig. 3, is considered to be infinitesimally thin, yet impose a finite mass per area on the resonator surface. The layer must be sufficiently thin and rigid so that a shear acoustic wave traversing the layer has a negligible phase shift; it moves synchronously with the crystal surface. The surface mechanical impedance is given by

$$
Z_{L}=j \omega \rho_{s}
$$

where $\rho_{s}$ is the mass per area of the layer. When Eq. (20) is substituted into Eq. (17) for the LEM, the resulting expression has only an imaginary component, meaning acoustic energy is stored and no power is dissipated. The frequency shift produced by the ideal mass layer is

$$
-\Delta f_{s} \cong-\frac{L_{2}}{2 L_{1}} f_{s}=-\frac{2 f_{s}^{2} \rho_{s}}{N \sqrt{\rho_{q} \mu_{q}}}
$$

which is equivalent to the Sauerbrey equation when $N=1[6]$. 
For mass layer sensors, the deviation between the resonator response predicted by the TLM and the LEM is small. In Fig. 4, the admittance magnitude and phase computed using Eq. (4) is plotted for the two models near the fundamental resonance frequency. The resonant frequency predicted by the LEM is $\sim 0.6 \%$ larger than that for the TLM, however, a large surface mass of $3 \mathrm{mg} / \mathrm{cm}^{2}$ is needed to create this difference. The frequency shift of both admittance curves is $\sim 161 \mathrm{kHz}$ from the $5 \mathrm{MHz}$ series resonance of the unperturbed resonator. Fig. 5 shows the frequency shift as a function of increasing surface mass density for the two models along with the prediction from the Sauerbrey Eq. (21). Deviations between the TLM and LEM are much smaller $\left(\sim 2.5 \%\right.$ at $\left.10 \mathrm{mg} / \mathrm{cm}^{2}\right)$ than deviations of either model from the Sauerbrey prediction $\left(\sim 19.5 \%\right.$ at $\left.10 \mathrm{mg} / \mathrm{cm}^{2}\right)$. The larger mass densities represented in Fig. 5 are unrealistically high for practical quartz resonator sensors; material volume densities would correspond only to the heaviest of elements and compounds, or layer thicknesses would exceed the limits of the negligible phase delay approximation.

\section{Liquid Loading}

A liquid at the surface of a quartz resonator is viscously-entrained as illustrated in Fig. 3. One-dimensional analysis of the fluid shear motion near the oscillating crystal surface is treated by White [7]. The resulting surface mechanical impedance for a Newtonian fluid is

$$
\mathrm{Z}_{\mathrm{L}}=(1+\mathrm{j}) \sqrt{\frac{\omega \rho \eta}{2}}
$$

where $\rho$ and $\eta$ are the liquid density and viscosity. For the LEM, Eq. (22) can be combined with Eq. (17), then reduced to give [4]

$$
R_{2}=\omega L_{2}=\frac{N \pi}{4 K_{0}^{2} \omega_{s} C_{0}} \sqrt{\frac{\omega \rho \eta}{2 \rho_{q} \mu_{q}}}
$$

Thus, for a Newtonian liquid, the power dissipation and energy storage components are equivalent, and both are proportional to $(\rho \eta)^{1 / 2}$. The frequency shift produced by the liquid load is

$$
\Delta f_{s}=-\frac{f_{s}}{2}\left[1+\frac{N}{2} \sqrt{\frac{\pi \rho_{q} \mu_{q}}{f_{s} \rho \eta}}\right]^{-1} \cong-\frac{f_{s}}{N} \sqrt{\frac{f_{s} \rho \eta}{\pi \rho_{q} \mu_{q}}} .
$$

This expression agrees with Kanazawa and Gordon [8]. 
Using the surface mechanical impedance for a liquid given by Eq. (23) in both the TLM and LEM allows for computation of differences between the models. Fig. 6 shows plots of the frequency shift and motional resistance change in the fundamental resonance of a liquid-loaded quartz resonator. The dashed line in each plot represents a linear response dependence on $(\rho \eta)^{1 / 2}$; the frequency shift is computed from Eq. (24). The largest value of $\rho \eta$ plotted in Fig. 6 is $1000 \mathrm{~g}^{2} \cdot \mathrm{cm}^{-4} \cdot \mathrm{s}^{-1}$ (a viscosity of $10^{5} \mathrm{cP}$ with $\rho=1 \mathrm{~g} / \mathrm{cm}^{3}$ ), which produces a deviation between the TLM and LEM of only $1.3 \%$. The deviation between models is slightly larger for $\Delta R$ compared to $\Delta \mathrm{f}_{\mathrm{s} .}$. As observed for the quartz resonator with a surface mass layer, the magnitude of liquid loading required to produce significant deviations between the TLM and LEM is beyond the practical limitation for a sensor.

\section{Viscoelastic Layers}

A quartz resonator can be used as a chemical sensor by depositing a thin layer on the surface. (See Fig. 7.) If the layer has an affinity for a target species, it will sorb the compound from the vapor or liquid phase changing the resonance characteristics of the system. The oscillating quartz crystal launches a shear acoustic wave into this surface film. In general, the film is viscoelastic and acoustically thick, so the shear wave will suffer loss and some phase shift as it propagates. The chemical sensor load layer is treated as a transmission line with non-piezoelectric properties as shown in Fig. 8. The transmission-line structure to the left in Fig. 8 is the quartz crystal resonator from Fig. 1. The resonator sees a surface mechanical impedance, $\mathrm{Z}_{\mathrm{L}}$, now consisting of the film impedance plus a load impedance, $Z_{1}$, at the layer surface. This mechanical impedance is described by the expression [9]

$$
Z_{L}=Z_{0} \frac{Z_{1} \cosh \left(\beta h_{f}\right)+Z_{0} \sinh \left(\beta h_{f}\right)}{Z_{0} \cosh \left(\beta h_{f}\right)+Z_{1} \sinh \left(\beta h_{f}\right)}
$$

where $Z_{0}=\left(\rho_{\mathrm{f}} G\right)^{1 / 2}$ is the film characteristic impedance, $\beta=j \omega\left(\rho_{\mathrm{f}} / \mathrm{G}\right)^{1 / 2}$ is the complex wave propagation constant, $h_{f}$ is the film thickness, $\rho_{f}$ is the film density, and $G=G^{\prime}+j G^{\prime \prime}$ is the film complex elastic modulus.

\section{Finite Film Only}

If the sensor viscoelastic layer is unloaded or tension-free at one surface, $Z_{1}=0$, Eq. (25) reduces to [5]

$$
Z_{\mathrm{L}}=j \sqrt{\rho_{\mathrm{f}} \mathrm{G}} \tan \left[\omega \sqrt{\frac{\rho_{\mathrm{f}}}{G}} h_{\mathrm{f}}\right] .
$$


This impedance cannot be decomposed into closed analytical forms for $L_{2}$ and $R_{2}$, so empirical evaluation is needed for both the TLM and LEM to compute frequency shifts and motional resistance changes. Eq. (26) contains four parameters $\left(G^{\prime}, G^{\prime \prime}, \rho_{f}\right.$, and $\left.h_{f}\right)$ that can vary for chemical sensor films, each affecting the resonator surface mechanical impedance. Some asymptotic conditions exist that simplify computations. In the limit of a thin rigid film, $G \rightarrow G^{\prime}$, and Eq. (26) reduces to Eq. (20) for an ideal mass layer. Conversely, at the other extreme is a film with all viscous behavior, $G \rightarrow j G^{\prime \prime}=j \omega \eta$ (Newtonian fluid); the layer appears semi-infinite, $h_{f} \rightarrow \infty$, to the acoustic wave, and Eq. (26) reduces to Eq. (22) for liquid loading. Initial observations then assume that viscoelastic layers would act like mass loads, liquid loads, or some intermediate viscous load all showing close agreement between TLM and LEM response predictions. However, missing from Eqs. (20) and (22) are phase shifts and interference effects that occur when shear waves propagate in finite thickness films. Including these effects, the resonator response can exhibit film resonance: that condition where the shear acoustic phase shift across the film, $\phi=\omega h_{f} \operatorname{Re}\left[\left(\rho_{i} / G\right)^{1 / 1}\right]$, is equal to odd multiples of $\pi / 2[5]$. Near film resonance, the surface mechanical impedance becomes large, and significant uncertainties exist in Eq. (16).

Two hypothetical films with $\rho_{\mathrm{f}}=1 \mathrm{~g} / \mathrm{cm}^{3}$ were chosen for this study: one is rigid, $\mathrm{G}^{\prime} \gg \mathrm{G}^{\prime \prime}$, and one exhibits loss, $G^{\prime} \approx G^{\prime \prime}$. During model computation, the film thickness, $h_{\mathfrak{f},}$ is varied so that resonator response always passes through the first film resonance. Fig. 9 shows the model-predicted shift in the fundamental series frequency versus film thickness for the rigid sensor layer $\left(G^{\prime}=10^{8}\right.$ dyne $/ \mathrm{cm}^{2}, G^{\prime \prime}=10^{3}$ dyne/cm $)$ on the crystal surface. The resonator exhibits a strong film resonance near $h_{f}=5 \mu \mathrm{m}$, where maximum frequency shifts are $\sim 280 \mathrm{kHz}$. Both the TLM and the LEM give similar results, with a maximum deviation of $\sim 0.5 \%$ between the models at the film resonance peak. Since the film is essentially lossless, the motional resistance shows no significant change at any film thickness. As the film thickness continues to increase, additional response (film) resonances are observed near $15 \mu \mathrm{m}, 25 \mu \mathrm{m}$, etc. each having identical characteristics to the one shown in Fig. 9.

Fig. 10 shows the TLM and LEM predictions for the lossy sensor film $\left(\mathrm{G}^{\prime}=10^{9}\right.$ dyne $/ \mathrm{cm}^{2}, \mathrm{G}^{\prime \prime}=3 \times 10^{8}$ dyne $/ \mathrm{cm}^{2}$ ). Plotted are the fundamental series resonance frequency shift (top) and motional resistance shift (bottom). Film resonance is again observed near a film thickness of $16 \mu \mathrm{m}$ in Fig. 10. The change in motional resistance near this resonance is quite significant $(\Delta R \sim 130 \mathrm{k} \Omega)$ and the maximum deviation between the model predications occurs here $(\sim 0.8 \%)$. When $\Delta f_{3}$ is maximum, the prediction deviations between models are extremely 
small, $<0.1 \%$. Relative differences between the models are greatest near the zero crossing of $\Delta f_{\mathrm{s}}$, but this is due to a small denominator in Eq. (18). Expected errors in using the LEM to extract sensor parameters (such as the layer thickness) near film resonance would be quite small.

\section{Film with Liquid Overlayer}

One advantage of quartz resonators is their ability to act as chemical sensors in a liquid environment. Analytes. sorbed from water or other fluids directly into a surface coating can be detected by the changes they create in the system resonance characteristics. Such a composite load is represented acoustically by a fixed thickness viscoelastic layer contacting the surface of the resonator with a semi-infinite liquid overlayer in contact with the opposite film surface. The surface mechanical impedance at the resonator surface looking into the film is given by Eq. (25) with $Z_{1}$ now described by Eq. (22) for the liquid. Resonator responses as predicted by the TLM and LEM are plotted in

Fig. 11 for a lossy viscoelastic layer with $\mathrm{G}^{\prime}=10^{9}$ dyne $/ \mathrm{cm}^{2}, \mathrm{G}^{\prime \prime}=3 \times 10^{8}$ dyne $/ \mathrm{cm}^{2}$, and $\rho_{\mathrm{f}}=1.5 \mathrm{~g} / \mathrm{cm}^{3}$, and a liquid overlay with $\rho \eta=0.01 \mathrm{~g}^{2} \cdot \mathrm{cm}^{-4} \cdot \mathrm{s}^{-1}$ (water or similar). Both the frequency shift (top plot in Fig. 11) and the motional resistance change (bottom) in the fundamental series resonance show the typical film resonance behavior as a function of film thickness. The film properties appear to dominate the resonator response such that the load imparted by the liquid has little influence except at the smaller thicknesses. For this composite load system, the agreement between the two computational models is quite good. Maximum deviation between the two models occurs at the peak of the $\Delta \mathrm{R}$ curve in Fig. 11 and is approximately $0.8 \%$.

The resonant response shifts versus film thickness illustrated in Fig. 11 can also be associated with electrodeposition of polymers from solution [10]. In such a system, an electroactive polymer film is deposited slowly on the resonator surface and the film thickness increases with time. Early in the process, mechanical loads are provided by the liquid; but as the film thickness increases, the resonator exhibits a response with more information about the viscoelastic layer. From the results of the comparative model investigations performed here, it is evident that such electrochemical studies can be modeled using lumped-element equivalent circuit representations and parameters of the polymer film extracted from impedance analysis of the system.

\section{Bounds on Surface Mechanical Impedance}


Based on results of the comparative computations between the transmission-line and limped-element models, it is useful to empirically determine the validity of Eq. (16), the prime assumption distinguishing the models. Using the definitions given by Eqs. (18) and (19), a maximum tolerable deviation between the models is set at $1 \%$. When this deviation limit is exceeded, it is assumed the LEM is no longer a valid representation of the TLM. We inspect the ratio $\mid Z_{\mathrm{L}} / Z_{\mathrm{q}}$ at this limit.

For mass-loaded resonators operating at the fundamental resonance, the tolerance limit is reached when $\rho_{3} \sim 5$ $\mathrm{mg} / \mathrm{cm}^{2}$. At this mass density, $\Delta \mathrm{f}_{\mathrm{s}} \sim 280 \mathrm{kHz}$. Then from Eq. (20), the magnitude of the surface mechanical impedance, $\left|Z_{\mathrm{L}}\right|$, is $1.58 \times 10^{5} \mathrm{~g} \cdot \mathrm{cm}^{-2} \cdot \mathrm{s}^{-1}$. This produces a load impedance ratio, $\left|Z_{\mathrm{L}}\right| Z_{\mathrm{q}} \approx 0.17$. For liquid-loaded resonators, $1 \%$ deviations occur between the models at $\rho \eta \sim 1000 \mathrm{~g}^{2} \cdot \mathrm{cm}^{-4} \cdot \mathrm{s}^{-1}$ which gives a ratio of surface mechanical impedance to quartz impedance of $\sim 0.2$. From these results, a bound can be established at $\left|Z_{\mathrm{L}}\right| / \mathrm{Z}_{q} \leq 0.1$ (with a comfortable margin) where the LEM will always be an adequate representation for TSM resonator sensors with mass and liquid loading.

For resonators with viscoelastic layers utilized as chemical sensors, similar results are obtained. For the rigid layer response plotted in Fig. 9, a maximum deviation of $\sim 0.5 \%$ between the models occurs at film resonance. Substituting film values from this point into Eq. (26) leads to $\left|Z_{L}\right| / Z_{q} \approx 0.18$. Since Eq. (26) scales as $G^{3 / 2}$, layers with larger modulus materials will produce larger values of $\left|Z_{\mathrm{L}}\right|$, and subsequently, increases in the ratio $\left|Z_{\mathrm{I}}\right| / Z_{\mathrm{q}}$. It is not uncommon for impedance ratios to approach 1.0 for some high modulus films, and still not produce deviations between the TLM and LEM that exceed 1\%. In fact, the lossy layer computations plotted in Fig. 10 are such a case. A maximum deviation between models of $0.9 \%$ is observed near film resonance, where $h_{\mathfrak{f}} \sim 16 \mu \mathrm{m}$ and $\Delta \mathrm{f}_{\mathrm{s}}$ is $\sim 90 \mathrm{kHz}$. The computed impedance ratio is $\left|\mathrm{Z}_{\mathrm{L}}\right| / \mathrm{Z}_{\mathrm{q}} \approx 0.85$. If we set the same bound on the ratio at $\left|\mathrm{Z}_{\mathrm{L}}\right| \mathrm{Z}_{\mathrm{q}} \leq$ 0.1 for the viscoelastic film-coated resonators as for the mass and liquid loaded resonators, it is certain that $1 \%$ deviations between the TLM and LEM will not be exceeded. However, the quantity $[2 \cdot \tan (\alpha / 2)]$ in Eq. (16) is often much larger than one, especially for coated resonators operating near film resonance $\left(\Delta \omega_{\mathrm{s}} \rightarrow 0\right)$, and more relaxed tolerances can be accepted for $\left|Z_{\mathrm{L}}\right| \mathrm{Z}_{\mathrm{q}}$.

\section{Conclusions}


For most practical sensor applications, we have shown that a lumped-element model can be used to accurately represent the surface-loaded TSM quartz resonator instead of the more complex transmission-line model. Sensor configurations included pure mass loading, liquid loading by a Newtonian fluid, viscoelastic thin-film loading, and loading by a viscoelastic film plus a semi-infinite liquid. Computations were performed using both models to determine relative deviations in two measurable sensor parameters: shift in the series resonant frequency, $\Delta f_{3}$, and increase in motional resistance, $\Delta \mathrm{R}$. For operation at the fundamental series resonance, relative deviations between the TLM and LEM for all configurations do not exceed 3\% until surface loads become extremely large and are no longer practical for sensor implementation. One of the simplifying assumptions allowing the TLM to reduce to the LEM is the load surface mechanical impedance is small compared to the quartz shear impedance. It has been determined empirically that when the impedance ratio is $<0.1$, relative deviation between the model predictions always will be $<1 \%$. This quantitative value establishes a reasonable upper limit where the LEM can be used to rapidly analyze impedance or admittance data to understand the resonator-load interaction and extract sensor parameters.

\section{Acknowledgments}

The authors thank G. C. Frye of Sandia National Laboratories and Prof. Fabian Josse of Marquette University for helpful discussions on this topic. H. L. Bandey thanks the University of Leicester for a scholarship. This work was supported by the United States Department of Energy under Contract DE-AC04-94AL85000. Sandia is a multiprogram laboratory operated by Sandia Corporation, a Lockheed Martin Company, for the United States Department of Energy.

\section{DISCLAIMER}

This report was prepared as an account of work sponsored by an agency of the United States Government. Neither the United States Government nor any agency thereof, nor any of their employees, makes any warranty, express or implied, or assumes any legal liability or responsibility for the accuracy, completeness, or usefulness of any information, apparatus, product, or process disclosed, or represents that its use would not infringe privately owned rights. Reference herein to any specific commercial product, process, or service by trade name, trademark, manufacturer, or otherwise does not necessarily constitute or imply its endorsement, recommendation, or favoring by the United States Government or any agency thereof. The views and opinions of authors expressed herein do not necessarily state or reflect those of the United States Government or any agency thereof. 


\section{References}

[1] J. F. Rosenblum, Bulk Acoustic Wave Theory and Devices (Artech House, Boston, 1988).

[2] R. Lucklum, C. Behling, R. W. Cernosek, and S. J. Martin, "Determination of Complex Shear Modulus with Thickness Shear Mode Resonators," J. Appl. Phys. 30, pp. 346-356 (1997).

[3] V. E. Granstaff and S. J. Martin, "Characterization of a Thickness-Shear Mode Quartz Resonator with Multiple Nonpiezoelectric Layers," J. Appl. Phys. 75 (3), pp. 1319-1329 (1994).

[4] S. J. Martin, V. E. Granstaff, G. C. Frye, "Characterization of a Quartz Crystal Microbalance with Simultaneous Mass and Liquid Loading," Anal. Chem. 63, pp. 2272-2281 (1991).

[5] S. J. Martin and G. C. Frye, "Polymer Film Characterization Using Quartz Resonators," Proc. 1991 IEEE Ultrasonics Symp., pp. 393-398 (1991).

[6] G. Sauerbrey, "Verwendung von Schwingquarzen zur Wägung dünner Schechten and zur Mikrowägung," $Z$. Phys. 155, pp. 206-222 (1959).

[7] F. M. White, Viscous Fluid Flow (McGraw-Hill, New York, 1991).

[8] K. K. Kanazawa and J. G. Gordon, "Frequency of a Quartz Microbalance in Contact with Liquid," Anal. Chem. 57, pp. 1770-1771 (1985).

[9] S. Ramo, J. R. Whinnery, and T. Van Duzer, Fields and Waves in Communication Electronics (Wiley, New York, 1965).

[10] H. L. Bandey, M. Gonsalves, A. R. Hillman, and S. J. Martin, "Crystal Impedance Characterisation of NonRigid Coupled Electroactive Polymer Films," Digest, Acoustic Wave-Based Sensors Symp., ECS Fall Mtg., San Antonio, TX, p. 1144 (1996) 
Table I. Properties of AT-Cut Quartz

\begin{tabular}{|l|l|l|}
\hline$\varepsilon_{\mathrm{q}}$ & $3.982 \times 10^{-13} \mathrm{~A}^{2} \cdot \mathrm{s}^{4} \cdot \mathrm{g}^{-1} \cdot \mathrm{cm}^{-3}$ & permittivity \\
\hline$\eta_{\mathrm{q}}$ & $3.5 \times 10^{-3} \mathrm{~g} / \mathrm{cm} / \mathrm{s}$ & viscosity \\
\hline$\mu_{\mathrm{q}}$ & $2.947 \times 10^{11}$ dyne $/ \mathrm{cm}^{2}$ & shear elastic constant \\
\hline$P_{\mathrm{q}}$ & $2.651 \mathrm{~g} / \mathrm{cm}^{3}$ & density \\
\hline $\mathrm{K}_{0}{ }^{2}$ & $7.74 \times 10^{-3}$ & electromechanical coupling factor \\
\hline
\end{tabular}

Table II. Unperturbed Quartz Resonator Model Parameters

\begin{tabular}{|l|l|}
\hline $\mathrm{N}$ & 1 (fundamental) \\
\hline $\mathrm{f}_{\mathrm{s}}$ & $5 \mathrm{MHz}$ \\
\hline $\mathrm{h}_{\mathrm{q}}$ & $0.033 \mathrm{~cm}$ \\
\hline $\mathrm{C}_{0}$ & $4.2 \mathrm{pF}$ \\
\hline $\mathrm{C}_{\mathrm{p}}$ & $5.0 \mathrm{pF}$ \\
\hline $\mathrm{R}_{1}$ & $10 \Omega$ \\
\hline $\mathrm{C}_{1}$ & $26.35 \mathrm{fF}$ \\
\hline $\mathrm{L}_{1}$ & $38.45 \mathrm{mH}$ \\
\hline
\end{tabular}




\section{Figure Captions}

Fig. 1. The transmission line representation of a piezoelectric quartz resonator with one tension-free surface and one loaded surface. This schematic is based on a three-port Mason model [1].

Fig. 2. The modified Butterworth-Van Dyke equivalent circuit for a surface-loaded quartz resonator [4,5]. (a) $A$ single motional impedance element, $Z_{m}{ }^{2}$, is used in series with elements for the unperturbed resonator, and (b) the load impedance broken into motional inductance, $L_{2}$, and resistance, $R_{2}$.

Fig. 3. Cross-sectional view of the fundamental mode shear displacement in a quartz resonator with both mass and liquid loading on one surface. The rigid mass layer moves synchronously with the crystal surface; liquids are viscously entrained by surface motion.

Fig. 4. The admittance magnitude and phase near the fundamental resonant frequency of a quartz resonator with a $3 \mathrm{mg} / \mathrm{cm}^{2}$ mass layer. The solid curve is the prediction by the transmission-line model and the dashed curve is by the lumped-element model.

Fig. 5. The frequency shift in the series fundamental resonance versus surface mass density for the transmissionline model (solid line), the lumped-element model (points), and the Sauerbrey equation (dashed line)[6].

Fig. 6. Shifts in series resonant frequency (top) and motional resistance (bottom) versus square root of the liquid density-viscosity product for the transmission-line model (solid lines) and the lumped-element model (points). The dashed lines are the response shifts for a linear model.

Fig. 7. A cross-sectional view of a quartz resonator chemical sensor showing shear displacement in the quartz and a surface viscoelastic film. A phase shift occurs as the acoustic wave propagates in the sensing layer.

Fig. 8. The transmission line representation of a non-piezoelectric sensing film on the quartz resonator surface. The additional mechanical load, $Z_{L}$, can be produced by any of several materials at the film surface.

Fig. 9. The series resonance frequency shift versus viscoelastic film thickness for the transmission-line model (solid line) and the lumped-element model (open circles). The resonator response exhibits a film resonance near a thickness of $5 \mu m$ in this rigid layer $\left(G^{\prime}=10^{8}\right.$ dyne $/ \mathrm{cm}^{2}, G^{\prime \prime}=10^{3}$ dyne $\left./ \mathrm{cm}^{2}\right)$.

Fig. 10. The shifts in series resonance frequency (top) and motional resistance (bottom) versus viscoelastic film thickness for the transmission-line model (solid line) and the lumped-element model (open circles). The resonator. response exhibits a film resonance near $16 \mu \mathrm{m}$ in this lossy layer $\left(G^{\prime}=10^{9}\right.$ dyne $/ \mathrm{cm}^{2}, G^{\prime \prime}=3 \times 10^{8}$ dyne/cm $\left.{ }^{2}\right)$.

Fig. 11. The shifts in series resonance frequency (top) and motional resistance (bottom) versus viscoelastic film thickness for the transmission-line model (solid line) and the lumped-element model (open circles). The lossy. viscoelastic layer is similar to that in Fig. 10 with a liquid of density-viscosity $1.5 \mathrm{~g}^{2} \mathrm{~cm}^{-4} \cdot \mathrm{s}^{-1}$ contacting the top surface. A film resonance occurs near $13 \mu \mathrm{m}$. 


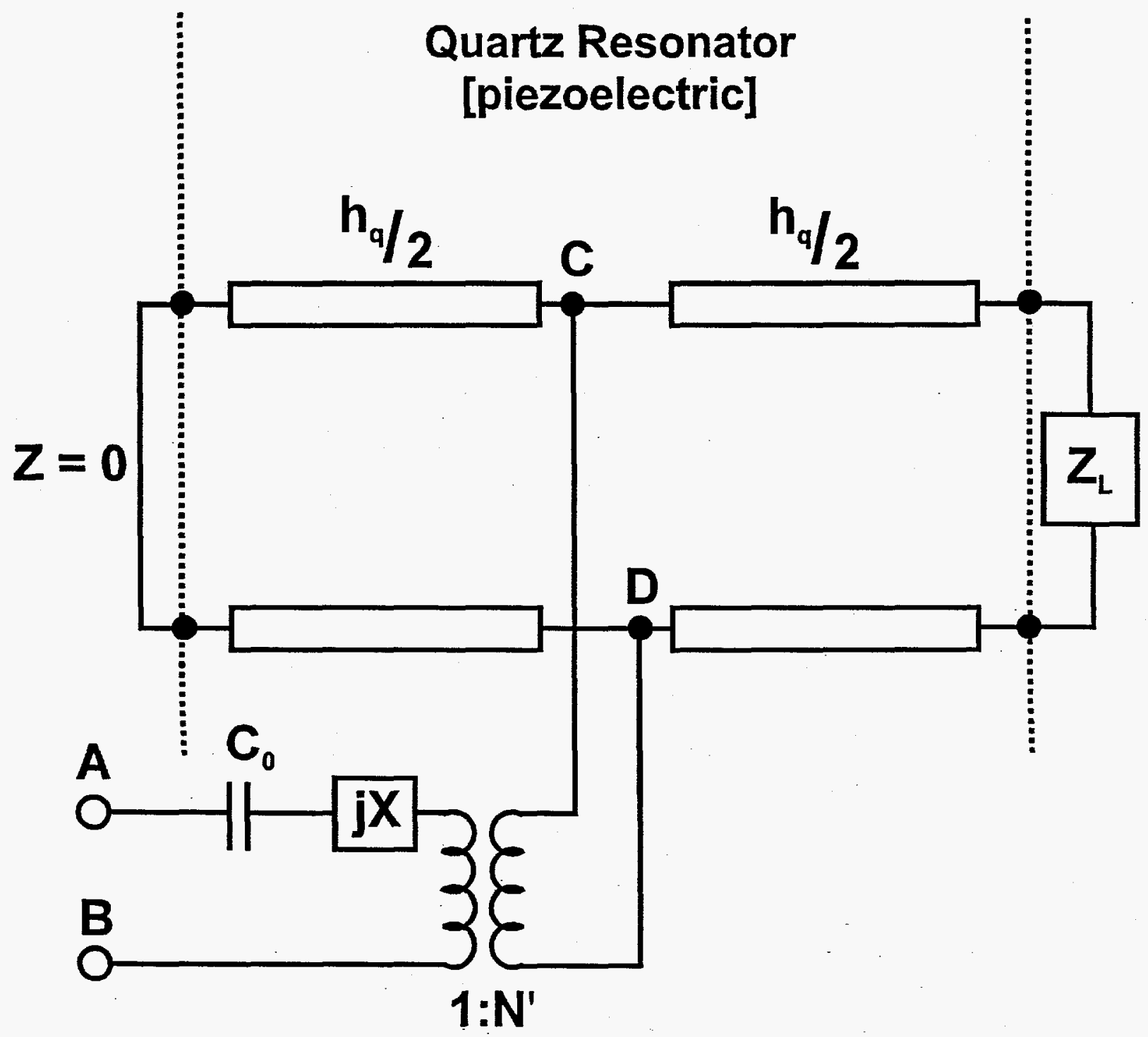




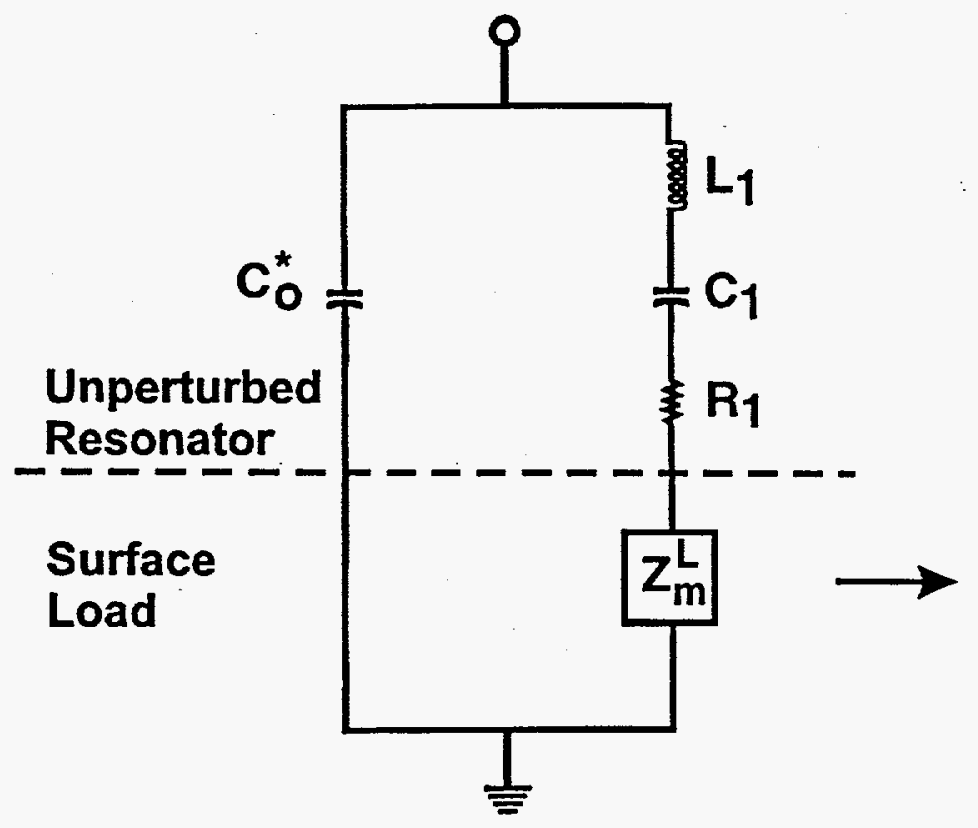

(a)

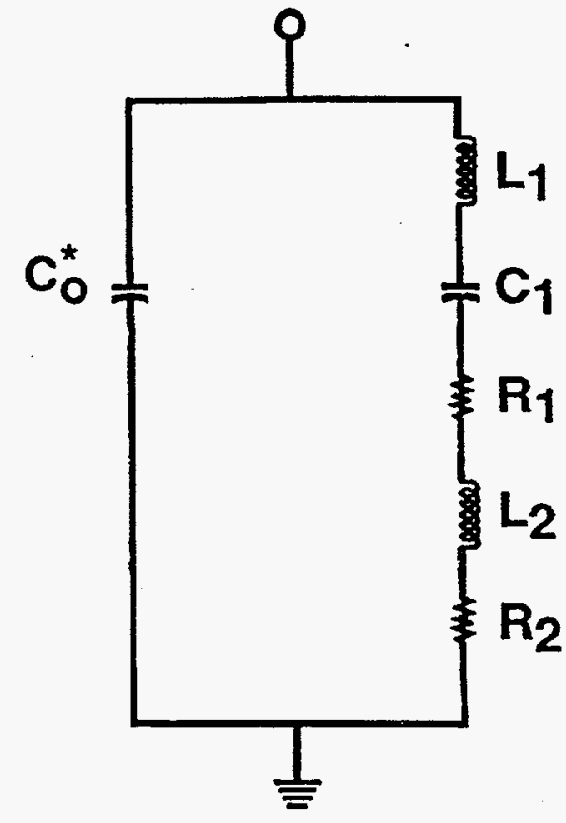

(b) 


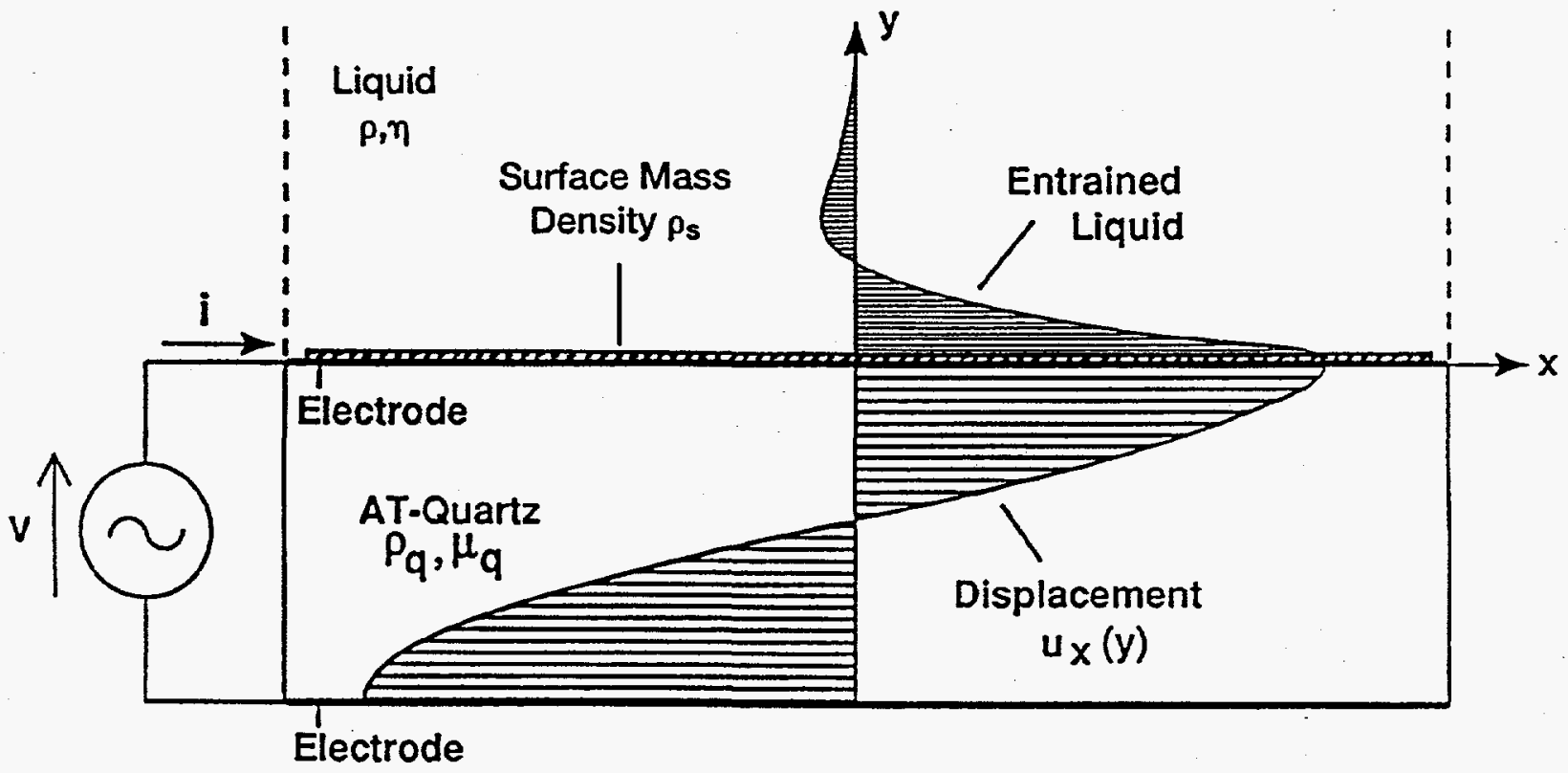




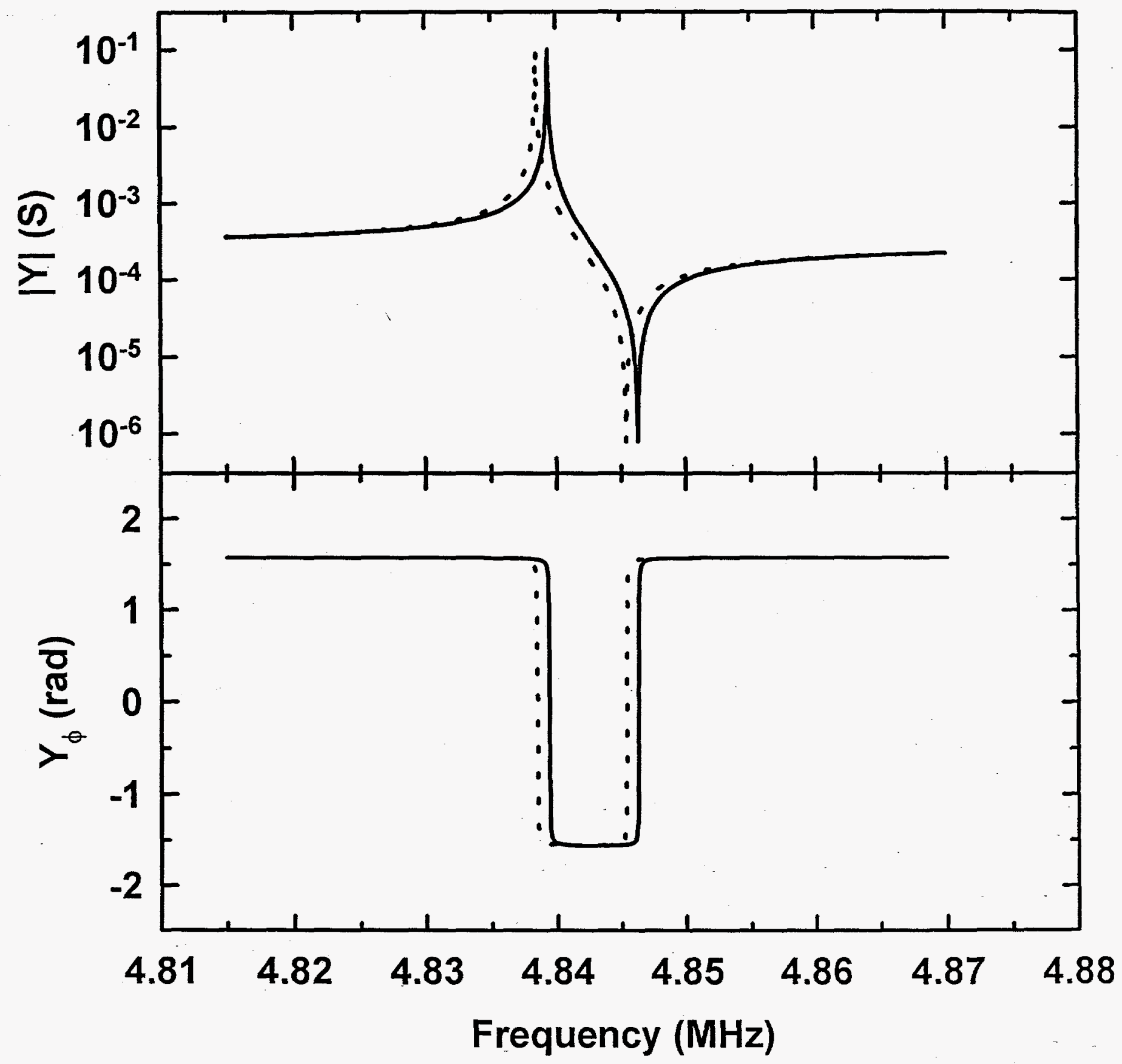




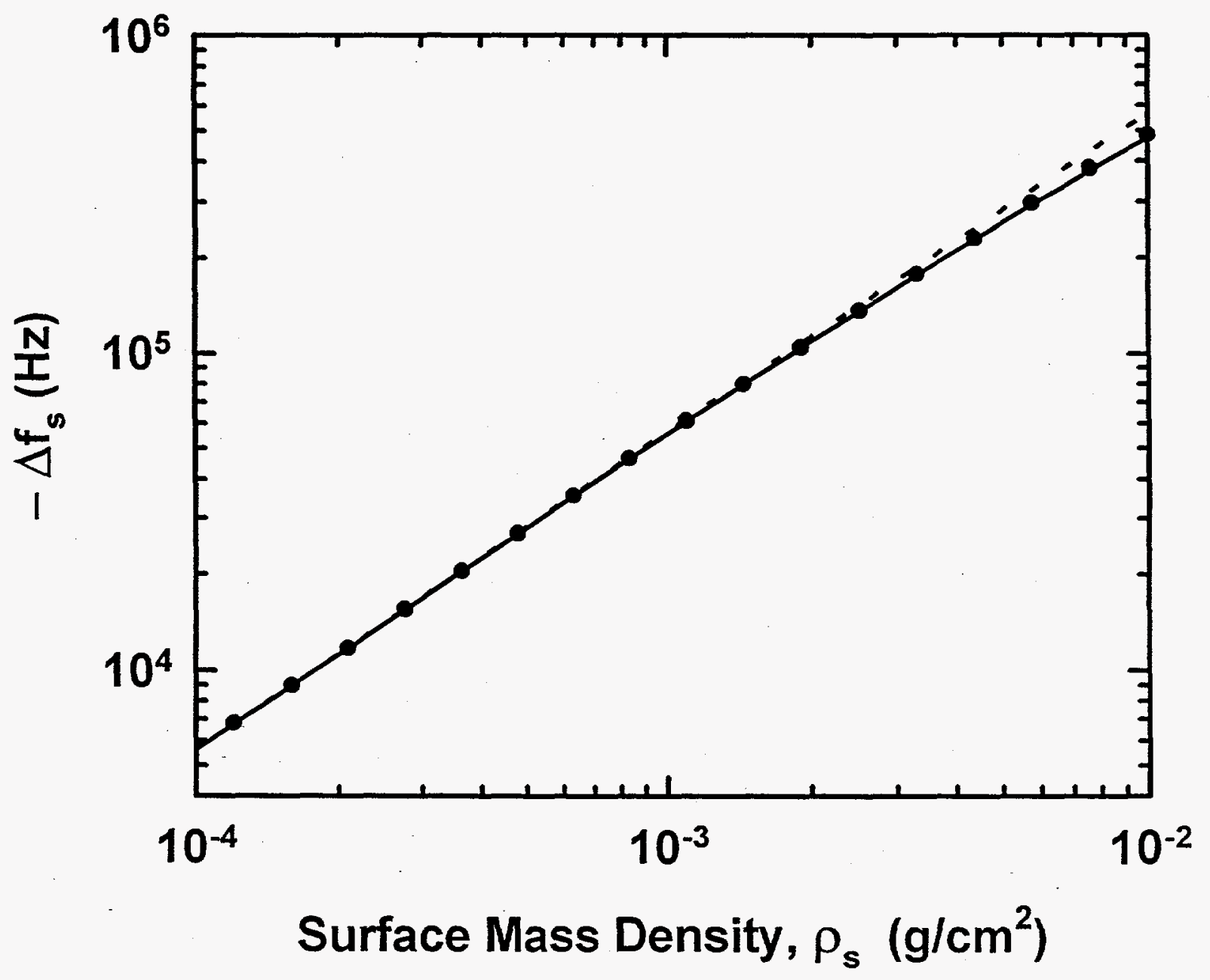




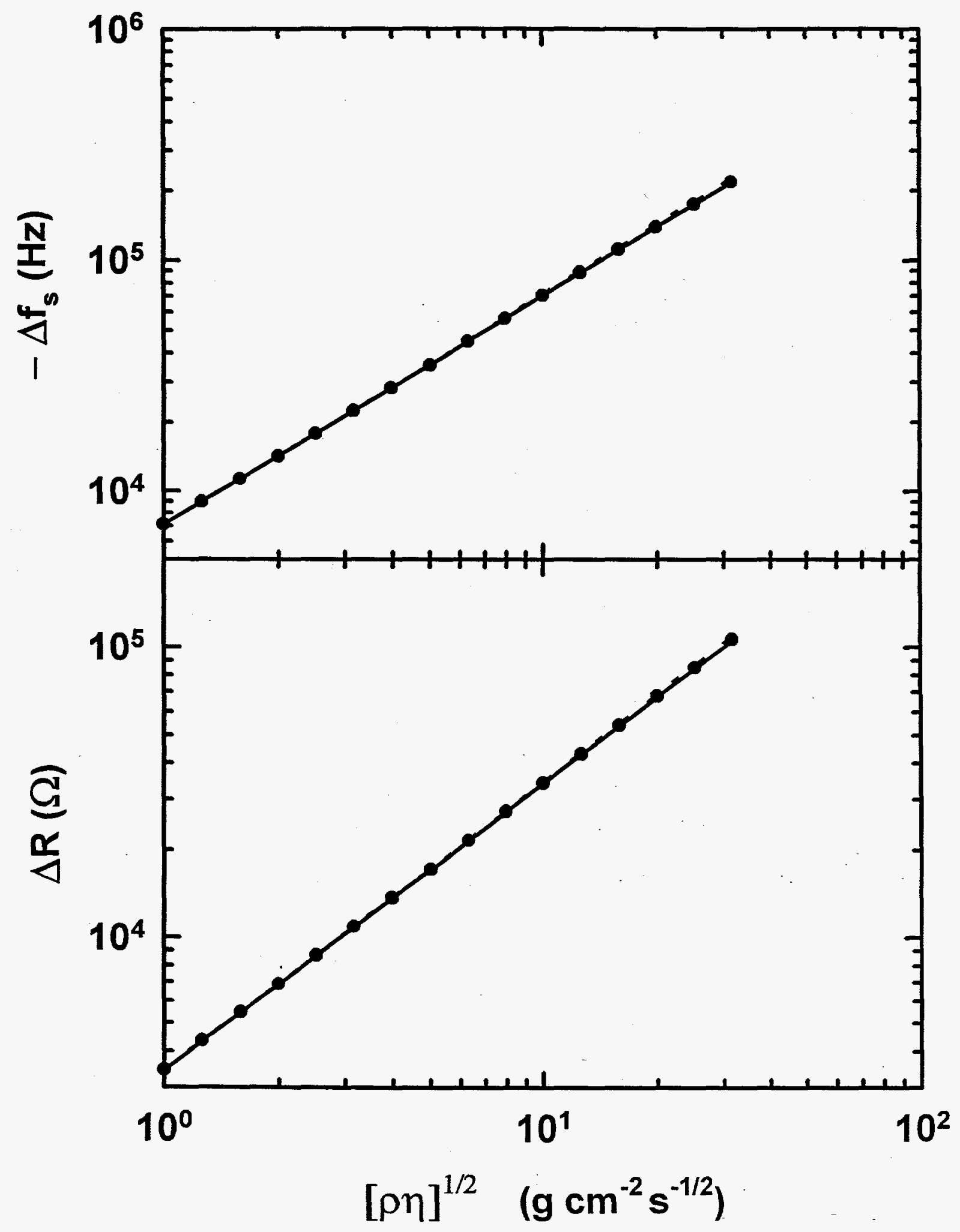




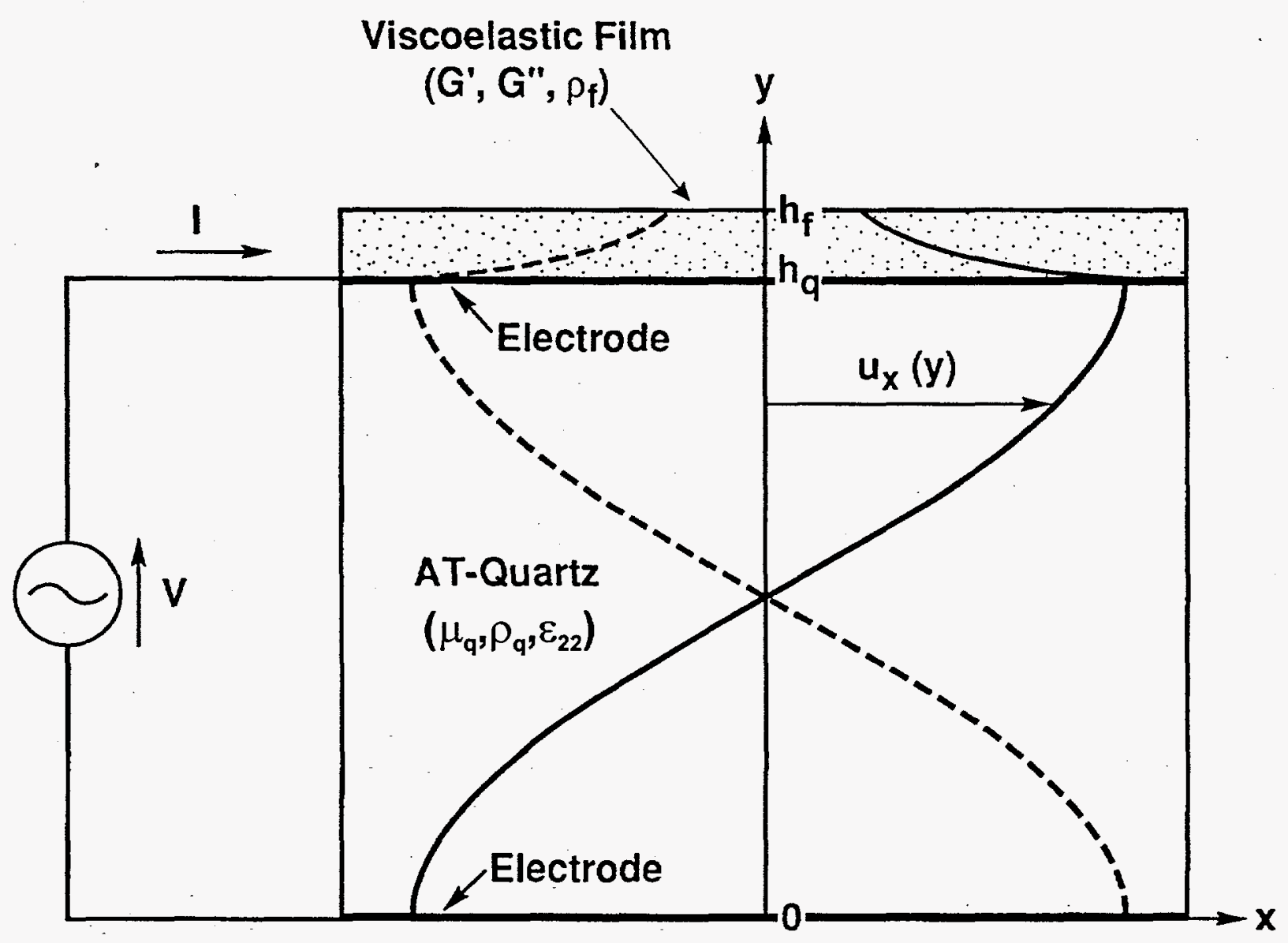




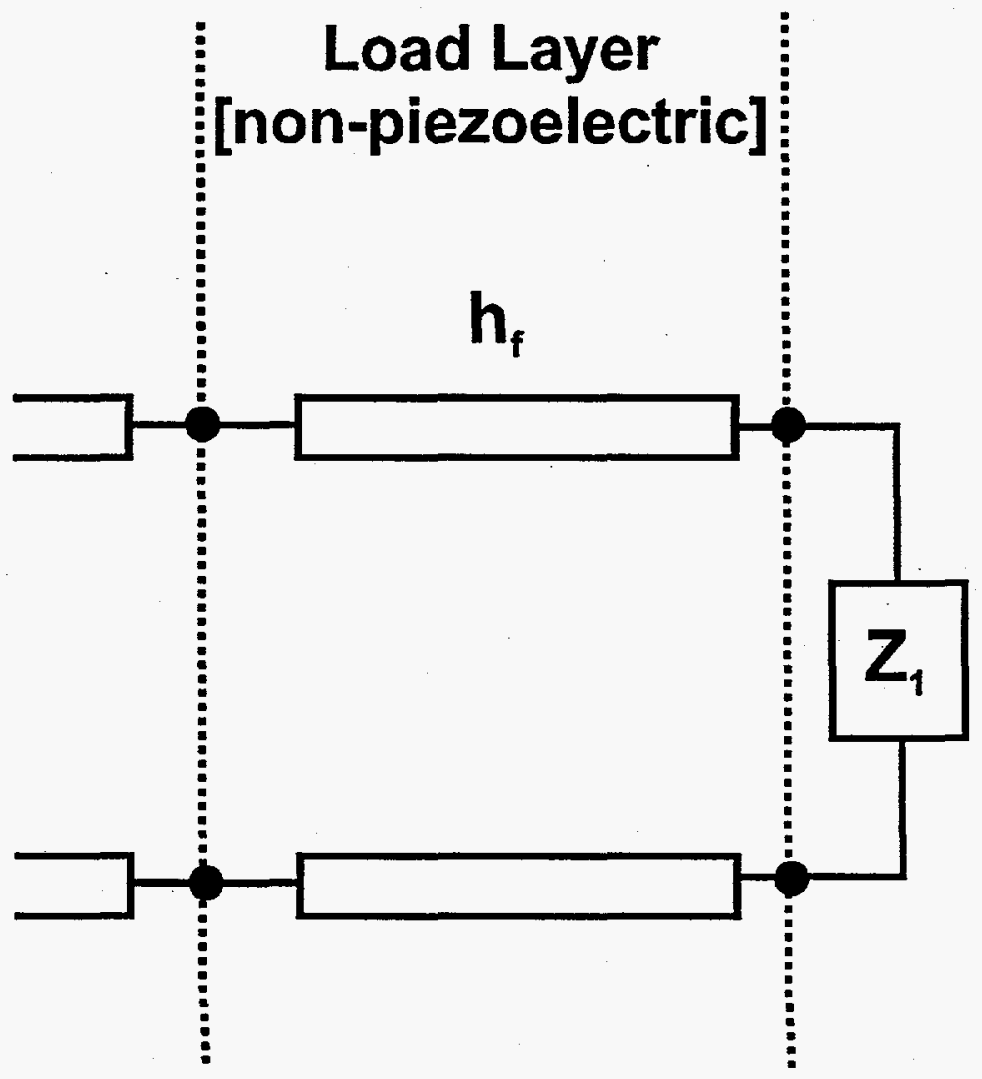




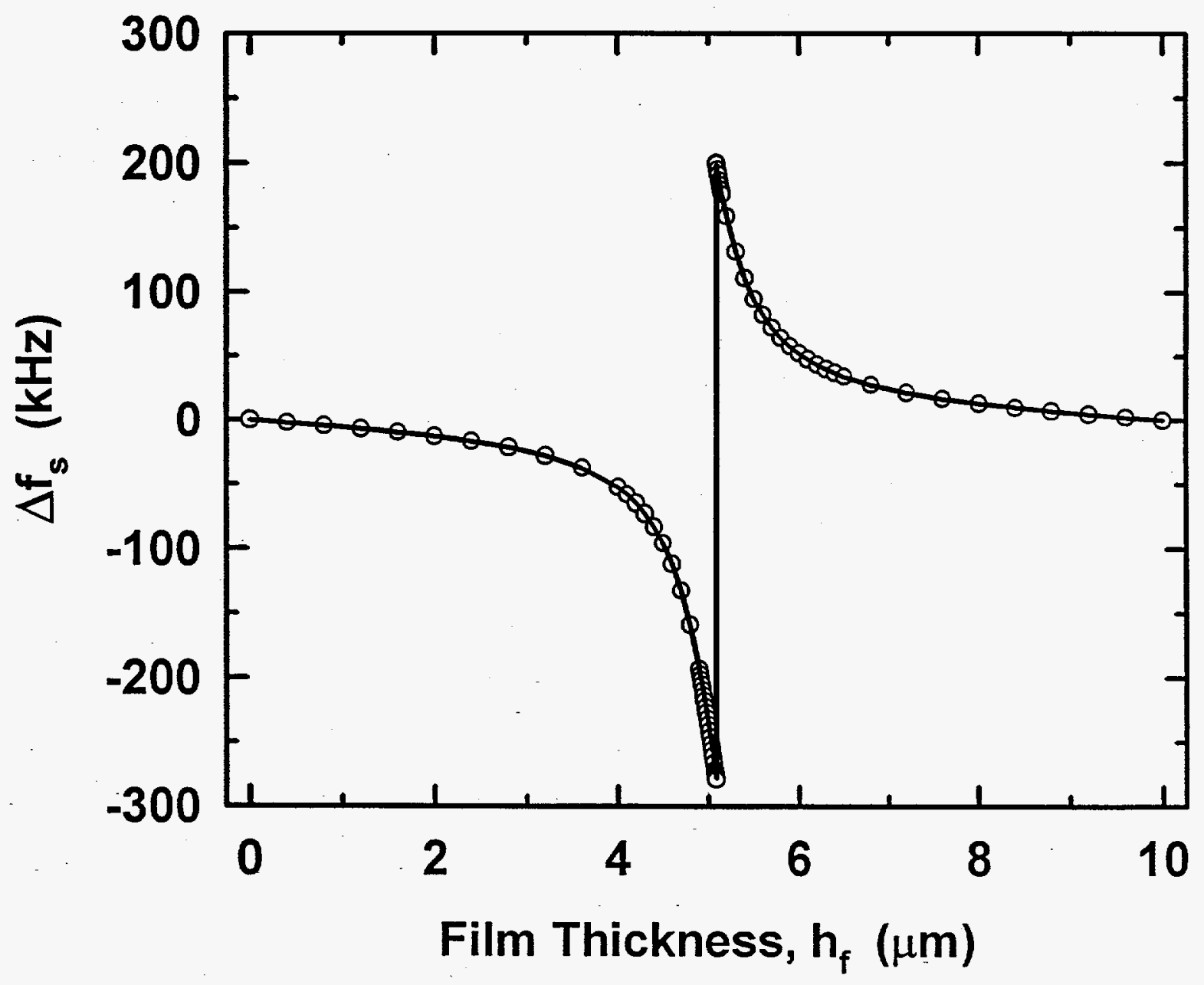




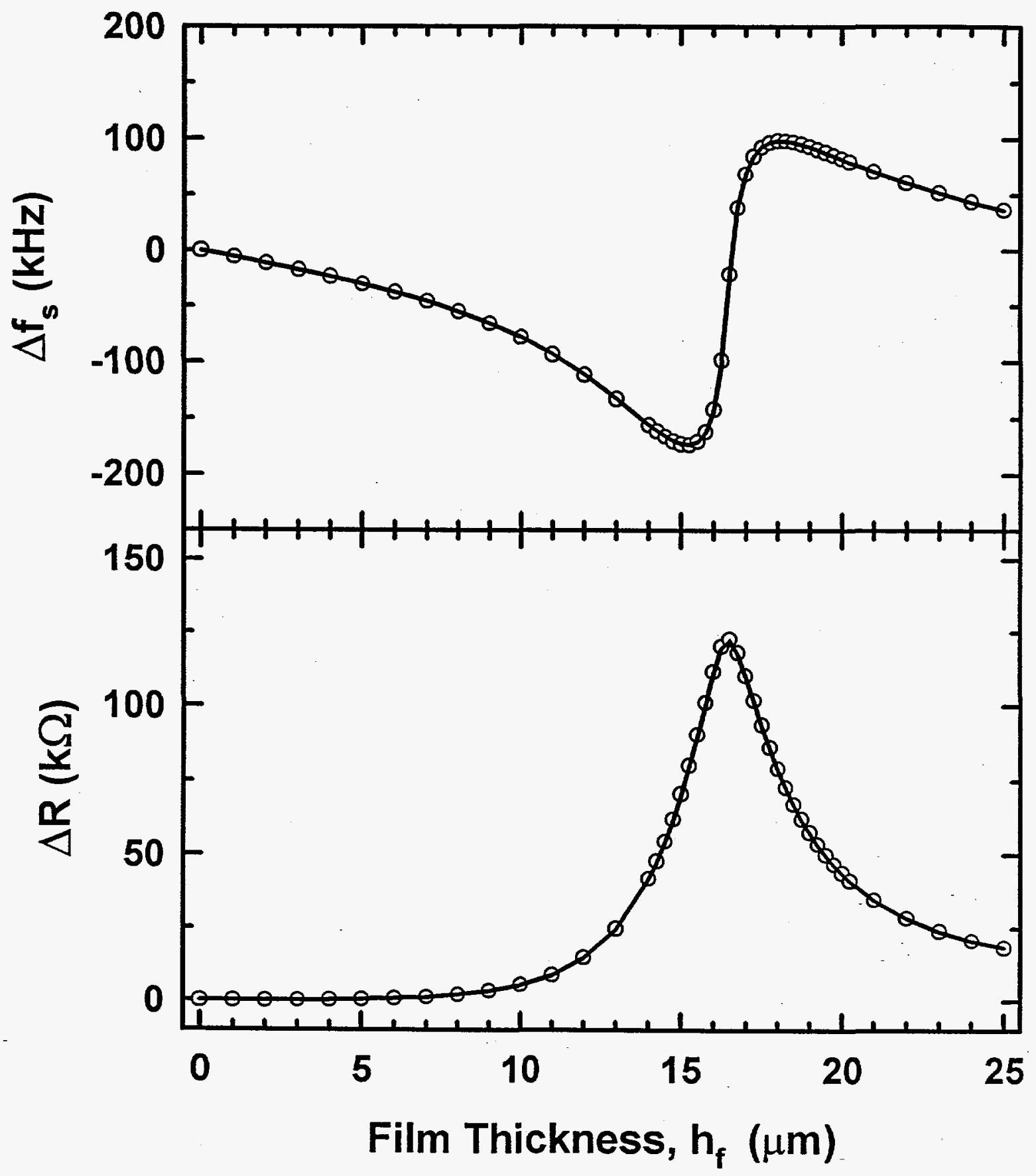




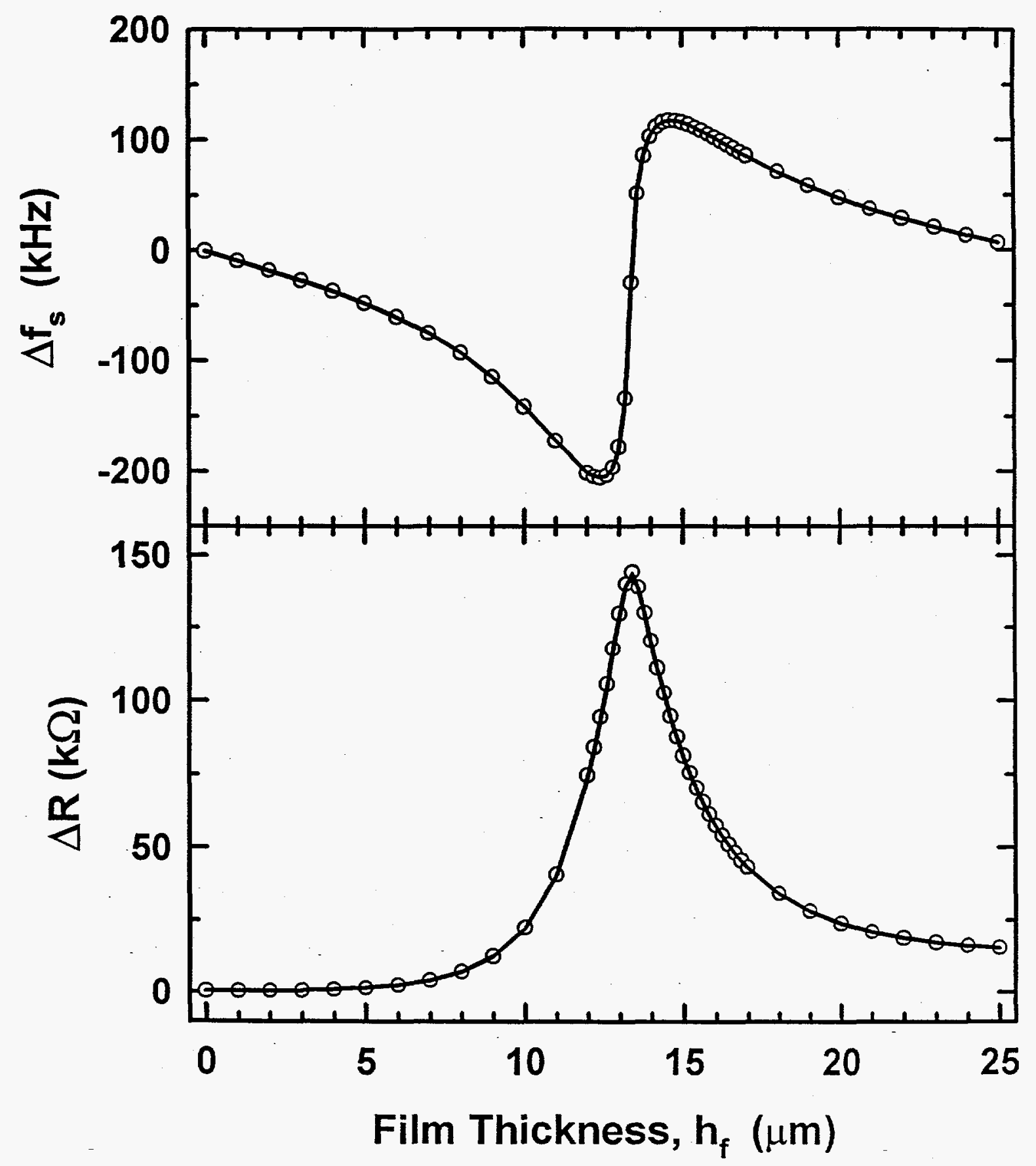

